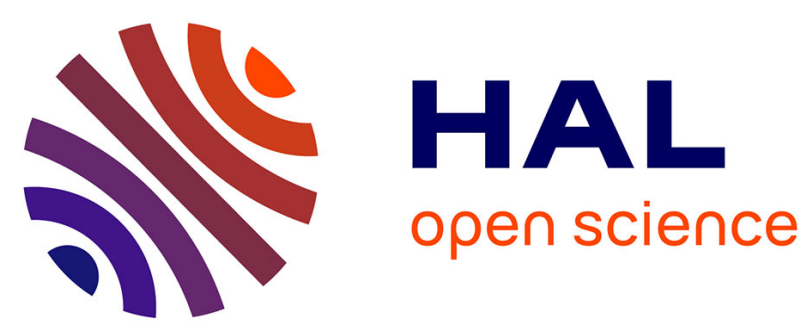

\title{
Adaptation of hybrid five-phase ABS algorithms for experimental validation
}

Mathieu Gerard, William Pasillas-Lépine, Edwin de Vries, Michel Verhaegen

\section{To cite this version:}

Mathieu Gerard, William Pasillas-Lépine, Edwin de Vries, Michel Verhaegen. Adaptation of hybrid five-phase ABS algorithms for experimental validation. AAC 2010, Jul 2010, Munich, Germany. pp.N/A. hal-00525927

\section{HAL Id: hal-00525927 https://hal.science/hal-00525927}

Submitted on 10 Jun 2013

HAL is a multi-disciplinary open access archive for the deposit and dissemination of scientific research documents, whether they are published or not. The documents may come from teaching and research institutions in France or abroad, or from public or private research centers.
L'archive ouverte pluridisciplinaire HAL, est destinée au dépôt et à la diffusion de documents scientifiques de niveau recherche, publiés ou non, émanant des établissements d'enseignement et de recherche français ou étrangers, des laboratoires publics ou privés. 


\title{
Adaptation of hybrid five-phase ABS algorithms for experimental validation
}

\author{
Mathieu Gerard* William Pasillas-Lépine ** \\ Edwin de Vries ${ }^{* * *}$ Michel Verhaegen* \\ * Delft Center for Systems and Control, Delft University of \\ Technology, Delft, The Netherlands (e-mail: m.p.gerard@tudelft.nl). \\ ** Laboratoire des signaux et systèmes (CNRS-Supélec), \\ Gif-sur-Yvette, France (e-mail: pasillas@lss.supelec.fr) \\ *** Engineering Dynamics, Delft University of Technology, Delft, The \\ Netherlands, (e-mail: e.j.h.devries@tudelft.nl)
}

\begin{abstract}
Anti-lock Braking System (ABS) is the most important active safety system for passenger cars, but unfortunately the literature is not really precise about its description, stability and performance. This research improves a five-phase hybrid algorithm based on wheel deceleration and validate it on a tyre-in-the-loop laboratory facility. Five relevant effects are modelled so that the simulation matches the reality. Two methods to deal with the time delays are proposed. It can be verified that the limit cycle of the ABS encircle the optimal braking point without having any tyre parameter a priori known.
\end{abstract}

Keywords: Anti-lock braking, Hybrid control systems, Limit cycle analysis, Experimental validation, Quarter-car.

\section{INTRODUCTION}

Anti-lock braking system (ABS) is the most important active safety system for road vehicles. There are two reasons for implementing an ABS system in a car. The potential of the tyre to generate lateral forces is decreased when the longitudinal slip is increased. The first ABS objective is therefore to limit the longitudinal slip in order to maintain steerability and lateral stability during heavy braking. Furthermore, the brake force generated by the tyre presents a maximum at a moderate slip. Maintaining the maximum brake force to reduce the brake distance is the second objective.

According to the Bosch website, 76 percent of all new vehicles were equipped with $\mathrm{ABS}$ in 2007 and it is now a standard equipment for passenger cars in the EU, the U.S. and Japan. This system has been around for more than 30 years since its introduction by Bosch in a Mercedes in 1978.

Unfortunately, it is still very difficult to find much details in the literature about the ABS algorithms used in practice. The algorithms found in Kiencke and Nielsen (2000) and Robert Bosch GmbH (2003) are based on heuristics and rules, and do not allow a systematic analysis. This for several reasons. First, it is difficult to understand why they work. Secondly they rely on a extensive tuning, which might be extremely difficult and costly to obtain since the influence of each parameter on the global performance is not clear. And finally, they require a lot of testing since no theory can help assessing e.g. the stability.

Recently, a five-phase hybrid algorithm has been studied by one of the authors. Details can be found in Pasillas-
Lépine (2006). Using a very basic quarter car model, the proposed algorithm was theoretically studied and the limit cycle was analysed. Conclusions could be drawn on how to tune the algorithm to achieve the best limit cycle and on the resulting performances. The objective of this work is to take the algorithm a step further and to implement it on a practical tyre-in-the-loop experimental setup at Delft University of Technology.

The simplified model used in Pasillas-Lépine (2006) is presented in Section 2. The modelling of the relevant phenomena for experimental implementation is described in Section 3. The theoretical algorithm derived in PasillasLépine (2006) is recalled in Section 4 and the systematic tuning is addressed. The influence of the time delay on the brake actuation being very important, the theoretical algorithm had to be adapted and two modifications are described in Section 5. Results from the experimental validation are analysed in Section 6, where limit cycles close to the ones expected from theory and simulation are obtained.

\section{SIMPLIFIED MODELLING}

The development of the five-phase algorithm presented in Section 4 is based on a simplified one-wheel model. Only the longitudinal dynamics of a single loaded wheel is considered. Even though weight transfer and combined slip are ignored, all the basic phenomena related to ABS already appear in this very simple model. Moreover, the limit cycles predicted by this model (Figure 3 ) are already quite close to those obtained with more realistic models (Figure 4) or in experiments (Figure 5 and 6). 


\subsection{Wheel speed}

The angular velocity $\omega$ of a given wheel of the vehicle has the following dynamics:

$$
I \dot{\omega}=-R F_{x}+T,
$$

where $I$ denotes the inertia of the wheel, $R$ its radius, $F_{x}$ the longitudinal tyre force, and $T$ the torque applied to the wheel.

The torque $T=T_{e}-T_{b}$ is composed of the engine torque $T_{e}$ and the brake torque $T_{b}$. We will assume that during ABS braking the clutch is open and thus neglect the engine torque. Moreover, we will assume that the brake torque is given by

$$
T_{b}=\gamma_{b} P_{b},
$$

where $P_{b}$ denotes the brake pressure and $\gamma_{b}$ the brake efficiency.

\subsection{Tyre force}

The longitudinal tyre force $F_{x}$ is often modelled by a relation

$$
F_{x}\left(\lambda, F_{z}\right)=\mu(\lambda) F_{z} .
$$

That is, by a function that depends linearly on vertical load $F_{z}$ and nonlinearly on wheel slip $\lambda$

$$
\lambda=\frac{R \omega-v_{x}}{v_{x}},
$$

where $v_{x}$ is the longitudinal speed of the vehicle. It should be noted that this definition of slip shows a singularity at zero vehicle speed.

The nondimensional tyre characteristic $\mu(\cdot)$ is a skewsymmetric curve, such that $\mu(0)=0$ and $\mu^{\prime}(0)>0$ (see Figure 1). It is important to stress that this curve presents a maximum at $\lambda=\lambda^{*}$ so that $\mu^{\prime}\left(\lambda^{*}\right)=0$.

Several mathematical formulas have been used to describe this curve. Pacejka (2006) uses trigonometric functions, Burckhardt (1993) exponentials, and both Kiencke and Nielsen (2000) and Pasillas-Lépine (2006) a second order rational fraction:

$$
\mu(\lambda)=\frac{a_{1} \lambda-a_{2} \lambda^{2}}{1-a_{3} \lambda+a_{4} \lambda^{2}}, \quad \text { for } \lambda \leq 0 .
$$

The important point is that all this mathematical formulas use coefficients that depend on tyre characteristics, road conditions, tyre pressure, temperature, etc. In order to prove the robustness of the ABS algorithm, these coefficients should be assumed to be unknown.

\subsection{Wheel acceleration}

The vehicle will be supposed to brake with the maximal constant deceleration $a_{x}^{*}$ allowed by road conditions, which is $a_{x}^{*}=-\mu\left(\lambda^{*}\right) g$. In other words

$$
\dot{v}_{x}=a_{x}^{*} \text {. }
$$

Moreover, the tyre load $F_{z}$ will be assumed to be constant.

If we define the wheel slip offset and the wheel acceleration offset by

$$
\begin{aligned}
& x_{1}=\lambda-\lambda^{*} \\
& x_{2}=R \dot{\omega}-a_{x}^{*},
\end{aligned}
$$

we obtain the following system:

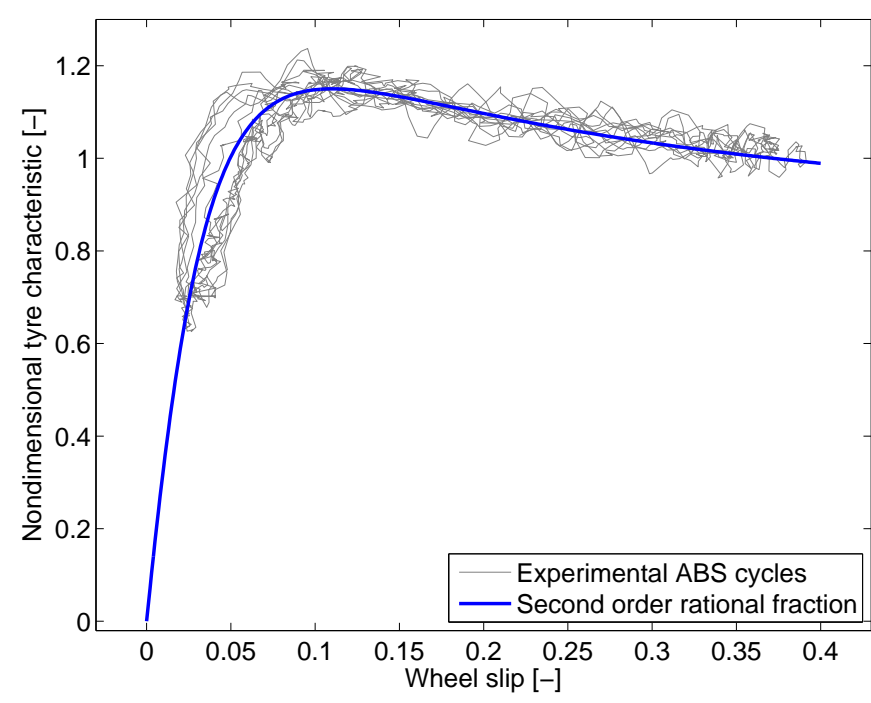

Figure 1. Dynamic tyre characteristic during an ABS braking with the controller of Section 5.1.

$$
\begin{aligned}
& \dot{x}_{1}=\frac{1}{v_{x}}\left(x_{2}-\left(\lambda^{*}+x_{1}\right) a_{x}^{*}\right) \\
& \dot{x}_{2}=-\frac{a}{v_{x}} \bar{\mu}^{\prime}\left(x_{1}\right)\left(x_{2}-\left(\lambda^{*}+x_{1}\right) a_{x}^{*}\right)+u,
\end{aligned}
$$

where

$$
a=\frac{R^{2}}{I} F_{z} \quad \text { and } \quad u=\frac{R}{I} \dot{T} .
$$

The function $\bar{\mu}(\cdot)$ is defined as

$$
\bar{\mu}(x)=\mu\left(\lambda^{*}+x\right)-\mu\left(\lambda^{*}\right),
$$

and thus represents the tyre characteristic with the peak at the origin.

\section{MODELLING PRACTICAL PHENOMENA}

The simplified model presented in Section 2 is not sufficient to explain the behaviour observed during experiments. The modelling of five additional effects is required.

\subsection{Oscillations in measurements}

When the tyre is rolling, oscillations can be seen in all measurement signals. Both longitudinal and vertical forces, and wheel speed and acceleration are perturbed. In our lab, such oscillations are periodic, with period equal to one wheel rotation. One plausible explanation comes from the difference in tyre properties along its circumference. Possible variations in wheel diameter, rubber carcas/beltstiffness, tread wear, etc. can lead to load oscillations. On a real vehicle, the road irregularity will also contribute to the load variation, resulting in a more random nature of the oscillations.

Such oscillations need to be taken into account since they can trigger control intervention at inappropriate moments. The switch between the phases of the hybrid controller is based on thresholds $\epsilon_{i}$ on wheel acceleration, as described in Section 4. There are two ways to deal with these oscillations in our algorithm: the first is to take large enough thresholds so that only significant variations in the acceleration signal are detected; the second is to filter 


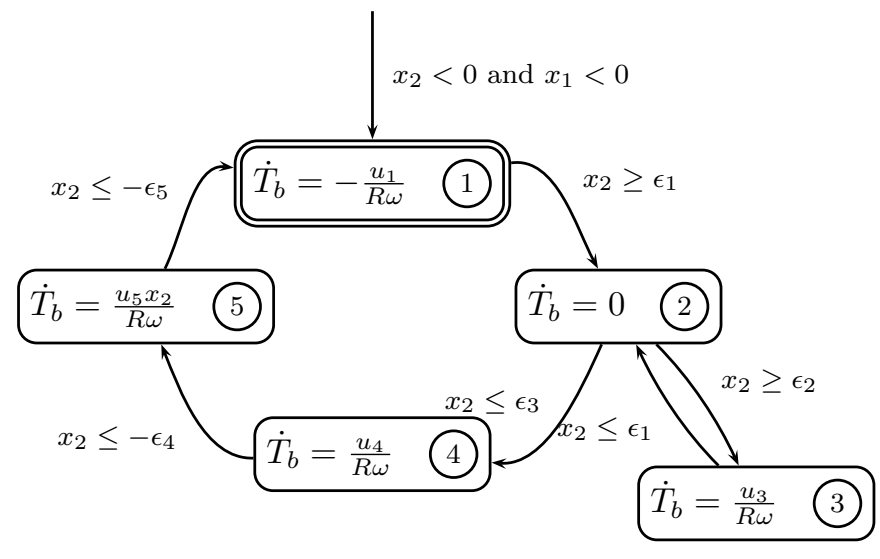

Figure 2. The five-phase ABS regulation logic.

the acceleration signal to remove undesired high frequent noise.

During testing, a compromise was chosen between both solutions. The acceleration signal is averaged on $1 / 10$ of the wheel rotation. At $18 \mathrm{~m} / \mathrm{s}$, the delay introduced due to filtering is about $5 \mathrm{~ms}$. With this filtering, peak to peak oscillations of $4 \mathrm{~m} / \mathrm{s}^{2}$ can still been observed. Therefore, a separation of $20 \mathrm{~m} / \mathrm{s}^{2}$ was taken between the thresholds $\epsilon_{i}$ to avoid mistriggering.

\subsection{Wheel Acceleration}

The wheel acceleration signal is not directly available and should be computed from the wheel angular encoder. The encoder signal consist of a series of pulses sent when one of the teeth of the encoder passes in front of the hall sensor. Linear regression on the raw encoder pulsed signal is used to fit a second-order curve to the time/displacement measurements. The second time derivative can then be calculated analytically, introducing much less noise than with numerical differentiation.

\subsection{Brake pressure dynamics}

The hydraulic line and the servo-valve used to control the pressure in the disk brake are limiting the performance of the actuation. In a first approximation, the controlled valve will introduce a second-order dynamics and a flow rate limit and the hydraulic line a transport time delay, between the desired and the actual brake pressure. Taking those effects into account is of first importance for designing a working ABS system.

A rough identification of the characteristics gives: a pure time delay of $7 \mathrm{~ms}$, a pressure rate limit between $+750 \mathrm{bar} / \mathrm{s}$ and $-500 \mathrm{bar} / \mathrm{s}$, and a second-order dynamics with a cut-off frequency of $60 \mathrm{~Hz}$ and a damping factor of 0.33 .

\subsection{Brake efficiency}

The brake efficiency $\gamma_{b}$ in Equation (2) is changing during braking depending on the brake temperature. In general, the efficiency will increase with the temperature, up to a certain level where fading starts and friction drops rapidly. This means that the ABS algorithm needs to be robust to changes in the brake properties. During a heavy braking on the test-bench, the gain $\gamma_{b}$ will typically increase by $30 \%$ in only 4 seconds.

\subsection{Relaxation length}

Work on tyre identification like Pacejka (2006) and Zegelaar (1998) has revealed that the force response of the tyre to various external inputs shows a lag in time. Because ABS controllers do give very abrupt commands to the brakes and to the tyre, such an effect absolutely needs to be taken into account. The fact that tyre deflections (of side walls, carcass and rubber thread) have to build up before the force is created calls for a model containing carcass compliance. A convenient method for modelling the transient behaviour is to filter the slip, see Zegelaar (1998):

$$
\sigma(\lambda) \dot{\lambda}+\left|v_{x}\right| \lambda=R \omega-v_{x}
$$

In Zegelaar (1998), the relaxation length $\sigma$ is found to decrease with the tyre slip $\lambda$, to finally reach zero at the top of the tyre characteristics. Due to the open-loop testing of tyre slip curves, Zegelaar obtained his results in the stable zone only.

The typical manifestation of the tyre relaxation effect can be seen on Figure 1. For the tyre in the stable zone $\left(\lambda<\lambda^{*}=0.1\right)$, the slip-force trajectory is not the same when increasing or decreasing the force. In particular, the external trajectory (on the left) will be followed during force decrease and the internal one when increased. In this work, the relaxation length at zero slip is roughly $\sigma(0)=0.1 \mathrm{~m}$ for the moderate normal load considered.

\section{THEORETICAL ALGORITHM}

For ABS regulation, the control objective is to keep the unmeasured variable $x_{1}$ in a small neighbourhood of zero, with a control $u$ that only uses the measured variable $y=$ $R \dot{\omega}-a_{x}^{*}$.

\subsection{The five-phase hybrid control strategy}

The ABS regulation logics borrowed from Pasillas-Lépine (2006) and studied in this paper is described on Figure 2. Each of the five phases of the algorithm define the control action that should be applied to the brake. As soon as one of the guard conditions that determines a discrete transition from the current phase becomes true, the algorithm will switch to the next phase and immediately change the control action. Note that this guard conditions only depend on the value of the wheel acceleration offset $x_{2}=R \omega-a_{x}$ which is measurable.

When a hybrid control law (based on discrete states) is used to control a continuous dynamical system, one obtains a hybrid automaton. We refer the reader to Lygeros et al. (2003) for a general description of these systems; and to Pasillas-Lépine (2006) for a detailed analysis of this specific ABS hybrid automaton. 


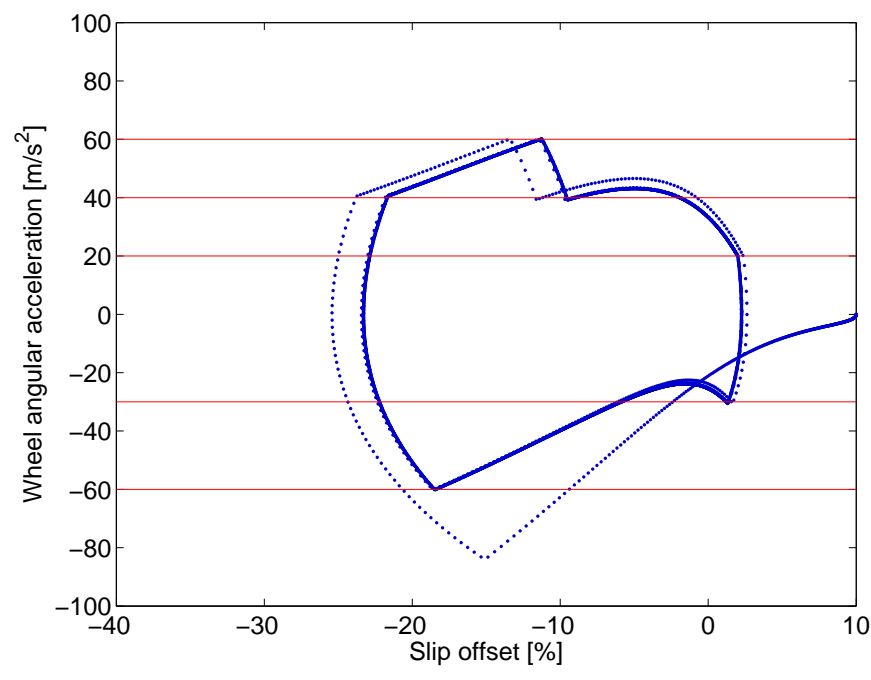

Figure 3. Limit cycle obtained when the theoretical fivephase algorithm of Section 4 is applied to the simplified model of Section 2.

\subsection{Tuning of the algorithm}

In order to tune the five-phase ABS algorithm, nine parameters need to be chosen: the wheel deceleration thresholds $\epsilon_{i}$ and the brake pressure derivatives $u_{i}$. The theoretical study of the limit cycles performed in PasillasLépine (2006) gives a good basis and clear rules to get an initial parameter set. The modelling of the experimental phenomena in Section 3 also allows for a systematic tuning.

Theoretically, the best performance is obtained taking symmetric thresholds : $\epsilon_{5}=\epsilon_{1}$ and $\epsilon_{4}=\epsilon_{3}$. Nevertheless, in practice, several phenomenon related to the delays impose taking slightly asymmetric thresholds in order to improve performance. Furthermore, the $\epsilon_{i}$ need to be separated with $20 \mathrm{~m} / \mathrm{s}^{2}$ as discussed in Section 3.1. In the experiments, the values $\epsilon_{1}=40, \epsilon_{2}=60, \epsilon_{3}=20$ and $\epsilon_{5}=30, \epsilon_{4}=50 \mathrm{~m} / \mathrm{s}^{2}$ were taken.

Ideally, the brake pressure variations should be as quick as possible in order to produce the smallest possible limit cycle. Therefore the maximum brake pressure derivatives allowed by the brake actuator were taken for the $u_{i}$ : $u_{1} / R \omega=500 \mathrm{bar} / \mathrm{sec}$ and $u_{i} / R \omega=750 \mathrm{bar} / \mathrm{sec}$ for $u_{3}$ and $u_{4}$, see Section 3.3.

The choice of $u_{5}$ is more delicate. If too small values of $u_{5}$ are taken, the cycle amplitudes are quite big and thus the performance is bad. If too big values of $u_{5}$ are taken, the algorithm becomes quite sensitive with respect to delays and the wheel might lock or remain caught in the stable zone of the tyre. A good compromise seems to be $u_{5} \frac{x_{2}}{R \omega}=50 \mathrm{bar} / \mathrm{sec}$, typically one order of magnitude less than quick pressure variations.

The limit cycle obtained with this specific tuning, when the five-phase algorithm is applied to the simplified model of Section 2 is shown on Figure 3.

\section{MODIFIED ALGORITHMS}

When implemented on the test bench, the theoretical version of the five-phase algorithm presented in Section 4

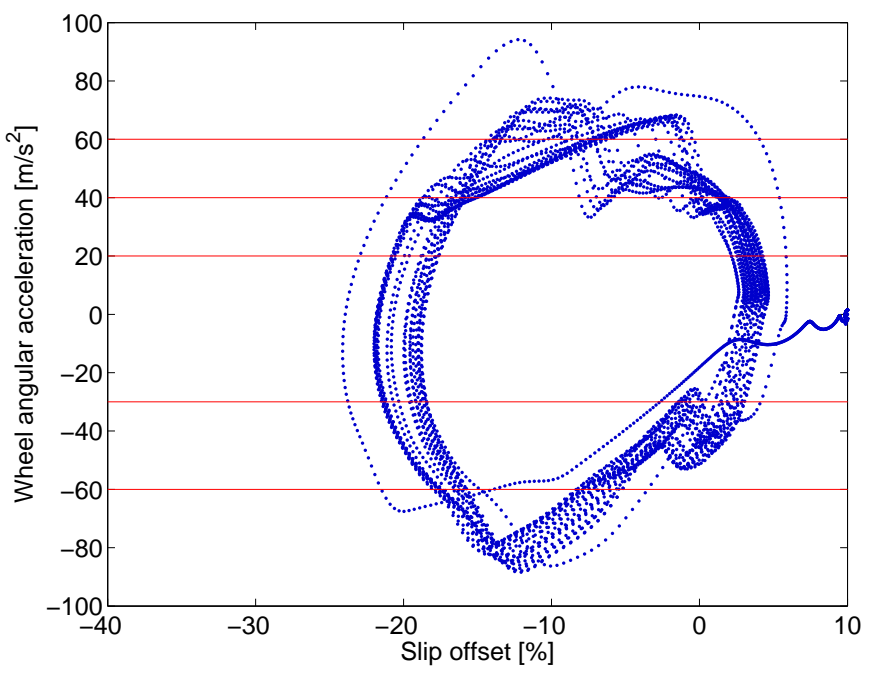

Figure 4. Limit cycle obtained when the modified algorithm of Section 5.2 is applied to a model that reproduces the issues of Section 3.

fails to cycle and remains blocked in an arbitrary phase. The delays, due to measurement filtering, tyre dynamics, and actuator limitations, have been identified to be the main cause of failure. Therefore, the theoretical algorithm need to be improved to be more robust to delays. Two methods are proposed and validated. All the delays are further lumped in a single global actuation delay.

\subsection{Pressure derivative profiles}

The first method to anticipate the delay is to slow down the pressure increase or decrease of phases 1,3 and 4 before the acceleration $x_{2}$ actually reaches the switching threshold $\epsilon_{i}$ (cfr. Figure 2). This could be equivalent to lowering the effective threshold $\bar{\epsilon}_{i}$ from which the pressure is kept constant. Because of the delay, the acceleration will continue evolving just until the original threshold $\epsilon_{i}$ is crossed. Unfortunately, the system uncertainties and the external disturbances will make the computation of $\bar{\epsilon}_{i}$ difficult, and since the system is quite sensitive, this will easily lead to failures. For this reason, the pressure derivative is kept non-zero until $\epsilon_{i}$ is reached, but is reduced as $x_{2}$ crosses $\bar{\epsilon}_{i}$. In that way, the system will be forced to cross $\epsilon_{i}$ ensuring the cycling, while the influence of the delay is minimized because of the low pressure derivative. Many pressure derivative profiles could be tried and compared. The following sigmoid is proposed:

$$
\dot{P}_{i}\left(x_{2}\right)=\frac{1}{2}\left(\dot{P}_{i}^{a}+\dot{P}_{i}^{b}\right)+\frac{1}{2}\left(\dot{P}_{i}^{b}-\dot{P}_{i}^{a}\right) \tanh \left(k_{i}\left(x_{2}-\bar{\epsilon}_{i}\right)\right) .
$$

where $\dot{P}_{i}^{a}$ and $\dot{P}_{i}^{a}$ are the brake pressure derivative at the maximal and minimal pressure derivatives during the phase. This sigmoid allows for a fast response of the system at the beginning of the phase while having a smooth transition at the end.

The main advantage of this approach is that it can compensate delays while remaining robust with respect to other effects like changes in brake efficiency. The main disadvantage is that this modified algorithm is more difficult to tune because it involves more parameters, that depend on the value of the delays and on vehicle velocity. 


\subsection{Open-loop pressure steps}

Another approach is to pre-compute, based on the wheel model (open-loop), the discontinuous brake pressure variation that will make the wheel go from the current acceleration to the desired one. This can be seen as the limit of the theoretical algorithm when all non-zero $u_{i}$ are taken infinitely big.

The brake pressure variation is given by :

$$
P\left(t_{0}^{+}\right)=P\left(t_{0}^{-}\right)+\frac{\zeta I}{R \gamma_{b}}\left(x_{2}\left(t_{0}^{+}\right)-x_{2}\left(t_{0}^{-}\right)\right) .
$$

where $t_{0}^{-}$and $t_{0}^{+}$are respectively the instants just before and just after the switch. For an ideal brake, no tuning parameter $\zeta$ is required. However in practice, asymmetry in the brake response requires small adjustments of $\zeta$. Further study of the brake will allow for a more systematic tuning. Also, real brake actuators are not able to follow such discontinuous pressure variations and a rate limit must be added.

The main advantages of this method is that it is intrinsically robust to any time delays and that it is closer to theory, and thus easier to tune and to understand. The limit cycles given by this controller, when practical phenomena are simulated, are shown on Figure 4. Moreover, it is better suited for brake actuators that follow a pressure or torque reference (instead of a pressure rate).

The main disadvantage is that it is less robust to other external disturbances like, in particular, brake efficiency variations (though it might be possible to estimate this unknown coefficient in real-time). Moreover, it might be cumbersome to implement on vehicles not equipped with brake pressure sensors.

\section{EXPERIMENTAL VALIDATION}

Both time delay anticipation methods presented above have been tested in practice and give the expected satisfying results. Thanks to the improved modelling, the experimental tests are very similar to the simulations. To limit the tuning effort, optimal performance was not the final aim and testing is done at constant vehicle speed.

\subsection{Experimental setup}

The tyre-in-the-loop experimental facility of Delft University of Technology on which the ABS is tested consist of a large steel drum of 2.5 meter diameter on top a which the tyre is rolling. An illustration can be seen on Figure 12.6 of Pacejka (2006). The setup has been used for many years for tyre modelling and identification using openloop excitation, see Pacejka (2006) and Zegelaar (1998). Recently, the electronics was upgraded in order to allow closed-loop tests to be performed and, in particular, rapid prototyping and testing of ABS strategies.

The wheel with the tyre is attached to an axle with a rigidly constrained height. The axle is supported by two bearings on both side of the wheel. The bearing housings are connected to the fixed frame by means of piezo-electric force transducers. An hydraulic disk brake is mounted on one side of the axle. The pressure in the calliper is locally controlled by a piece of analog electronics connected to a servo-valve, in order to match the reference pressure.

\subsection{Pressure derivative profiles}

Experimental testing shows that the ABS controller based on pressure derivative profiles works well in practice. Measurements are plotted on Figure 5. It can be noticed that, even with the large disturbance on the brake force and acceleration, the controlled system reaches and maintains a consistent limit cycle, encircling the optimal point $(0,0)$.

Because the reduction of the pressure derivative, when approaching the switching threshold, is probably not big enough yet, the time delay is still causing the acceleration to go far outside the predefined thresholds. Various tests have shown that the more positive the maximal acceleration, the larger the force drop in the stable zone. The difficulty to maintain the acceleration within the bounds is therefore responsible for the moderate performance of this algorithm regarding the predicted brake distance. Of course, a more precise tuning of the pressure derivative profile should solve this issue.

The longitudinal slip oscillates between 5 and 40 percent, which is good. It could be desired to have an even smaller excursion in order the maintain even more lateral stability and decrease brake distance. However, with this type of ABS methods based on wheel acceleration, the slip excursion needed to activate the phases is not directly controlled and depends heavily on the shape of the tyre curve. The larger the slopes of the tyre characteristic and the smaller the slip variation.

The tuning of this algorithm is quite robust. Small changes in the parameters or in the system do not affect the general shape of the limit cycle. Because the pressure is always kept decreasing or increasing respectively in phases 1 and 4, the acceleration will more robustly cycle between positive and negative and influences from vehicle speed or brake efficiency are minimized.

\subsection{Open-loop pressure steps}

The ABS controller based on open-loop pressure reference has also been successfully tested in practice. The measurements are plotted on Figure 6. Also in this case, the limit cycles are clearly visible and reproducible despite the disturbance.

This version is much better at maintaining the acceleration within the predefined thresholds. Intrinsically, the robustness to the time delay is larger. Thanks to the maximum acceleration maintained at a lower positive value, the force drop in the stable zone is smaller and the corresponding brake distance would also be reduced.

Unfortunately, since the pressure commands are given in open-loop, the algorithm has difficulties to react to system changes. In particular the tuning regarding the brake efficiency is quite sensitive. A too small gain could prevent from triggering the next phase, while a too large gain would make the acceleration to go far outside the thresholds, thus reducing the performance. Furthermore, the tuning of the phases 1 and 4 need to be different in practice compared to simulation in order to cope with the 

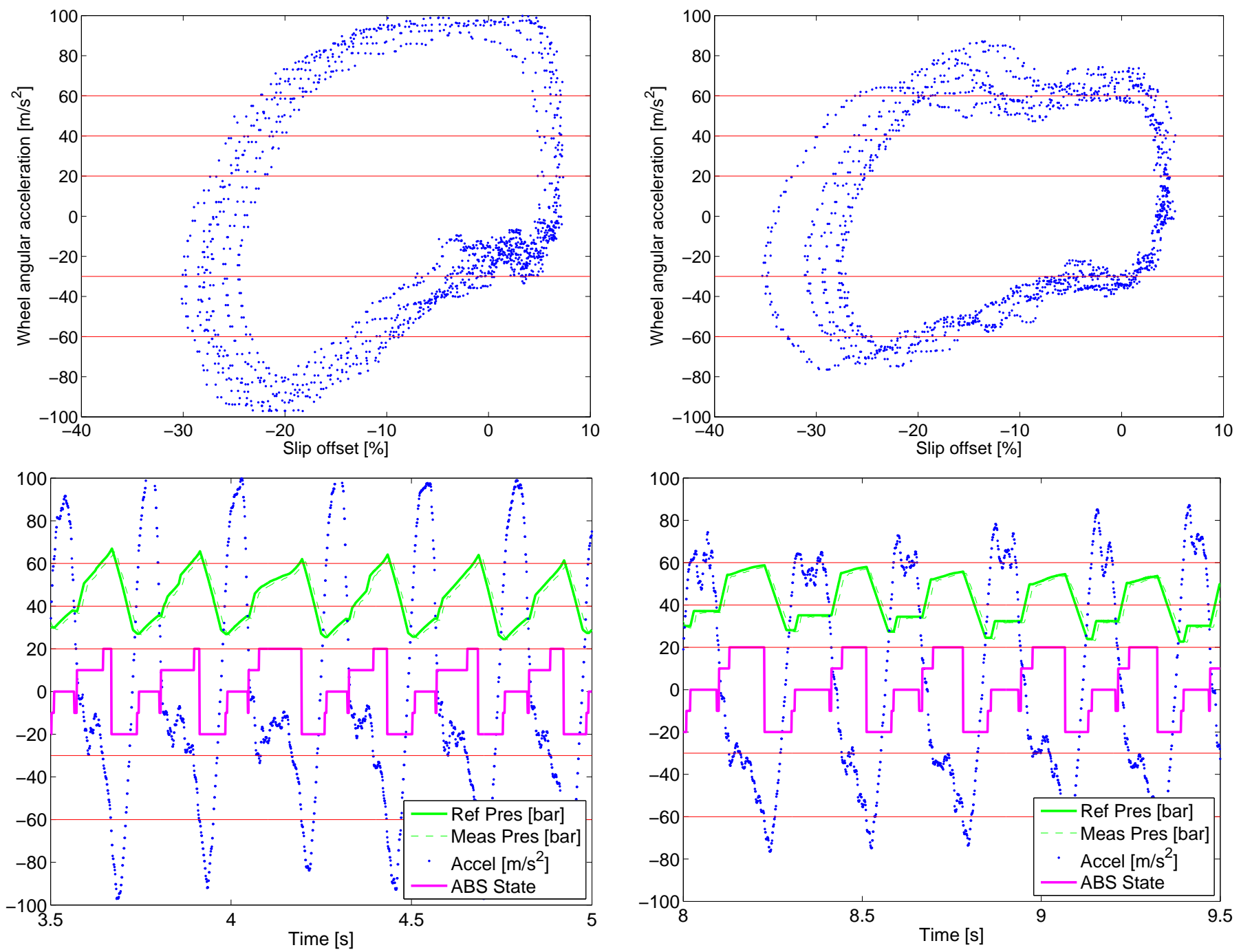

Figure 5. Experimental results for the ABS algorithm based on pressure derivative profiles.

encountered asymmetry in the brake reaction when increasing or decreasing the pressure. The robustness gained against the time delay seems to harm the robustness against other phenomena.

\section{CONCLUSION}

Time delays in measurement and actuation are the main reasons why the theoretical five-phase algorithm fails in experiments. Using pressure derivative profiles reduces the influence of delays, but does not perfectly compensate for them. This method is difficult to tune because of the large number of parameters involved, but maintains a good level of robustness with respect to other phenomena (like changes in brake efficiency). Giving pressure reference steps in open-loop offers more resistance with respect to time delays and remains closer to the original theoretical framework but requires a good estimation of brake efficiency. Both techniques give experimental limit cycles encircling the optimal braking point, without knowledge of tyre characteristics and only relying on wheel acceleration measurement, like predicted by the theory.

Figure 6. Experimental results for the ABS algorithm based on open-loop pressure references.

\section{REFERENCES}

Burckhardt, M. (1993). Fahrwerktechnik: Radschlupfregelsysteme. Vogel-Verlag, Germany.

Kiencke, U. and Nielsen, L. (2000). Automotive Control Systems. Springer-Verlag.

Lygeros, J., Johansson, K., Simić, S., Zhang, J., and Sastry, S. (2003). Dynamical properties of hybrid automata. IEEE Transaction on Automatic Control, 48(1), 2-17.

Pacejka, H.B. (2006). Tyre and Vehicle Dynamics. Butterworths-Heinemann, London.

Pasillas-Lépine, W. (2006). Hybrid modeling and limit cycle analysis for a class of five-phase anti-lock brake algorithms. Vehicle System Dynamics, 44(2), 173-188.

Robert Bosch GmbH (2003). Automotive Handbook. Bentley.

Zegelaar, P.W.A. (1998). The dynamic Response of Tyres to Brake Torque Variations and Road Unevenness. PhD Thesis, Delft University of Technology, Delft ISBN 90370-0166-1. 